\title{
BLOOD VESSEL DIAMETER MEASUREMENT ON RETINAL IMAGE
}

\author{
${ }^{1}$ Nidhal K. El Abbadi and ${ }^{2}$ Enas Al Saadi \\ ${ }^{1}$ Department of Computer Science, Education College, University of Kufa, Najaf, Iraq \\ ${ }^{2}$ Department of Computer Science, Education College, University of Babylon, Babylon, Iraq
}

Received 2013-08-15; Revised 2013-09-27; Accepted 2014-01-18

\begin{abstract}
Accurate measurement of vessel diameters on retinal images plays an important part in diagnosing cardiovascular diseases and early signs of certain systemic diseases, such as diabetes and hypertension. In this study we develop new method based on computer aided to determine the width of retinal blood vessels by analysing the color fundus image. Retinal vessel diameter was measured based on blood vessel wall estimating on the digital fundus image. We suggested a dependable and accurate technique to determine the diameter (width) of retinal blood vessel based on suggested mask created to use specifically on measuring the blood vessel diameter. The suggested system starts by removing the bifurcations and determine the width for each blood vessel segment. Suggested algorithm tested and gives accurate and promised results.
\end{abstract}

Keywords: Retina, Diameter, Blood Vessel, Image Processing, Diabetic

\section{INTRODUCTION}

Ocular fundus image can provide information on pathological changes due to some eye diseases and early signs of certain systemic diseases, such as diabetes and hypertension. The importants of ophthalmology diagnostic procedure by analyzing the fundus images become an important fields for researchers to automate this procedure.The corner stone in determine many diseases is by studying the structure of retinal blood vessels, which appear in the form of measurable abnormalities in diameter, color and tortuosity. Thus, there are great need for a reliable techniques to detecting the abnormalities in the blood vessels.

Retina images allow noninvasive viewing of the in-vivo vessels and have been established as indicator for incidence of diabetic retinopathy, early indicator of stroke and hypertension. It is the best modality to see the microvascular abnormalities such as change in the width of the vasculature. Changes in the width of the retinal arteriole and venues are known as direct indictors of retinal vasculature abnormality; detection of which requires accurate measurement of retinal vessel diameter. However, complex background and uneven lighting conditions result in poor contrast at vessel edges and this result in inaccurate diameter measurement (Kumar et al., 2012).

Complications of cardiovascular disease, such as stroke and myocardial infarction, have high mortality and morbidity. Determine the ratio of retinal vessel diameter of Arterial to Venous (AV) have important role for eye diseases and also can provide an important factor for an independant risks for stroke and myocardial infarct. In retinal images, the boundaries of the blood column form a reliable proxy for vessel diameter. Automated determination of the AV-ratio is therefore of high interest, but also complicated, because retinal vessel width and the contrast to the background vary greatly across retinal images (Xu et al., 2011).

The diabetic retinopathy can be indicated by observation the changing in the diameter of retinal blood vessels within the fundus image. One of the most powerfull sign of diabetic retinopathy is the unusual changing in the width along a vein. Generalized and focal retinal arteriolar narrowing and arteriovenous nicking have been shown to be strongly associated with current and past hypertension reflecting the transient and Corresponding Author: Nidhal K. El Abbadi, Department of Computer Science, Education College, University of Kufa, Najaf, Iraq 
persistent structural effects of elevated blood pressure on the retinal vascular network. In addition retinal arteriolar bifurcation diameter exponents have been shown to be changed significantly in patients with peripheral vascular disease and arteriosclerosis and a variety of retinal micro-vascular abnormalities have been shown to be related to the risk of stroke. Future precise retinal vascular analysis may allow risk stratification of a variety of arteriosclerotic diseases and clarify the microvascular contributions to clinical cardiovascular disease.

In all the above cases, it should be note that the interst is the changing in the width along the vessel and not the absolute diameter.

\section{RELATED WORKS}

Pemp et al. (2013) Introduced a method to measuring width of central retinal artery from noninvasive ocular hemodynamic measurements for diabetes patients. These diameters compared with blood vessels diameter of healthy peoples. The width of central retinal artery were calculated from retinal hemodynamic parameters measured with noninvasive measurement techniques.

An automatic vessel diameter measurement technique based on Linear Discriminate Analysis (LDA) has been proposed by (Kumar et al., 2012). After estimating the vessel wall, the vessel cross-section profile is divided into three regions: Two corresponding to the background and one to the vessel.

Klein et al. (2012) introduced a measurement of variations in retinal vessel width and incidence and progression of diabetic retinopathy, to describe the relationship of variation in retinal vessel widths to the subsequent 6-year incidence and progression of Diabetic Retinopathy (DR) and incidence of Proliferative Diabetic Retinopathy (PDR) and Macular Edema (ME) in persons with diabetes mellitus.

$\mathrm{Xu}$ et al. (2011) introduced amethod to segment the two blood vessel edges in fundus image using the graph-based algorithm to facilitate measuring the diameter of retinal blood vessels. First, the simultaneous two-boundary segmentation problem is modeled as a two-slice, 3-D surface segmentation problem which is further converted into the problem of computing a minimum closed set in a nodeweighted graph. An initial segmentation is generated from a vessel probability image.

Pakter et al. (2011) present a technique to determine the retinal vessel width with computer aided, but it is not clear which component of the vessels they are measuring. His paper was conducted to compare measurements of retinal vessel width by using imagingprocessing software on color Fundus Photographs (FPs) and Fluorescein Angiographs (FAs).

\section{METHODOLOGY}

This study focuses on how to find the blood vessels width in the retina image. Different clinical studies highlighted that the vessel width increases with wall shear stress and the vessel wall thickness increases with the circumferential wall stress (Joshi, 2012).

First step in this study is to localize and remove the nodes in the origin retinal binary image using techniques we suggested and published in other paper (El Abbadi and Al Saadi, 2013). All these nodes will be removed by changing the value of pixels around the nodes coordinates to zero value (black), we can imagine the detected nodes as a red circles drawing around the nodes (node is the center of circle) as shown in Fig. 1 (figure was hand drawing).

The diameter or width of blood vessel at a point in a binary image is defined as the shortest line segment (Euclidean distance) passing between two edges (blood vessel wall), points (A and B) as shown in Fig. 2.

By removing the nodes from blood vessels network we get segmented blood vessels network as shown in Fig. 3, every segment in the segmented blood vessels network image is labeled by image label algorithm, this can be accomplished by scan all the pixels in the image, the non zero pixels will be labeled as preliminary labels and recording label equivalences in a union-find table. The union-find algorithm (The Union-Find algorithm is used for maintaining a number of non-overlapping sets from a finite universe of elements) used to resolve the equivalence classes. Finally relabeled the pixels based on the resolved equivalence classes.

The interested blood vessel is selected and separated in new image to facilitate the computing of its diameter, always the interested vessel will be oriented horizontally in the new image. Computing its diameter start by passing mask (created for this study) on the image that contains blood vessel segment from upper to the lower in order to read pixels vertically, the created mask is shown in Fig. 4.

Value of the mask is computed by summation of multiplying each value of the mask elements by the corresponding pixel in the image. If the result equal to 3 that means pixel on the one edge of the blood vessel.

Coordinates of the blood vessel edge resulted from the previous operation will be stored (point A). 


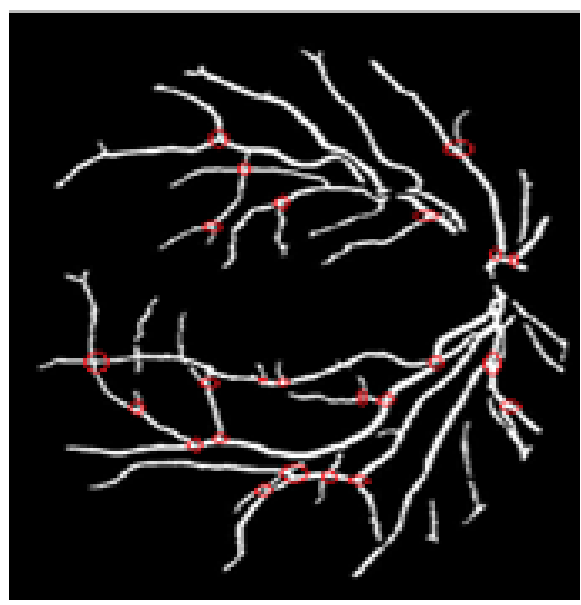

Fig. 1. Localizing of blood vessels networks nodes

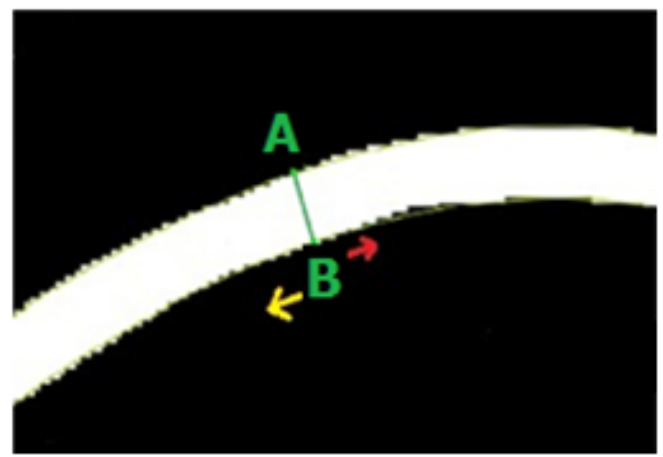

Fig. 2. Vessel width estimated as the euclidean distance between $\mathrm{A}$ and $\mathrm{B}$

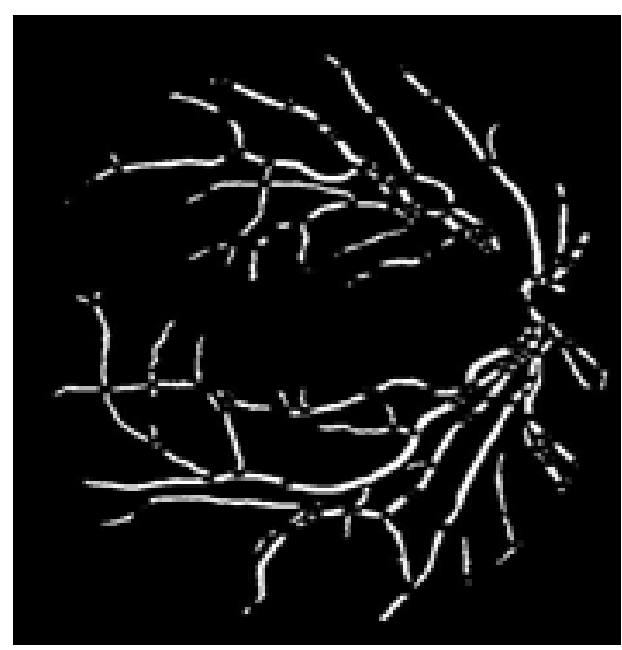

Fig. 3. Segmented blood vessels after removing nodes

$$
\left|\begin{array}{lll}
1 & 0 & 1 \\
0 & 1 & 0 \\
1 & 0 & 1
\end{array}\right|
$$

Fig. 4. Suggested mask

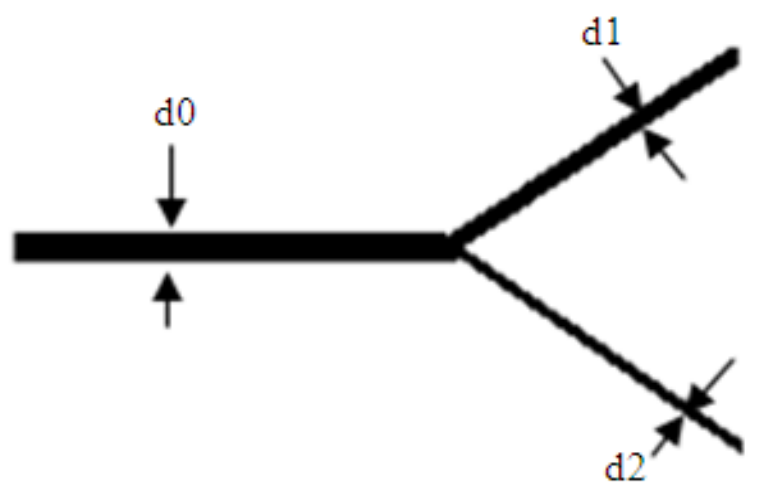

Fig. 5. Simulation of blood vessel with predefined diameter for test algorithm

The mask is continue passing from upper to the lower (moving one pixel at each step) and compute its value, if it is not 3 then continue moving toward the other end of the blood vessel, this process continue until get the mask value equal to 3 , this is the second point B as shows in (Fig. 2).

Distance between (A) and (B) is computed based on (Euclidean distance) and saved the result in variable named (Dis), the equation of The Euclidean distance (Dis) between two points A and B is:

$$
\text { Dis }=\sqrt{\left|X_{A}-X_{B}\right|^{2}+\left|Y_{A}-Y_{B}\right|^{2}}
$$

To get the shortest distance, point B (corresponding to the center of the mask) is move to the right (Fig. 2) one pixel (the mask shift to the right) and compute the value of mask, if the value not equal to 3 then the mask will move to the upper or to the lower one pixel in order to get 3 (this keep the center of the mask on the blood vessel edge), in this case, coordinates is determined (coordinate is determined always for the center of the mask).

Distance between (A) and new point is computed, if the distance is less than the previous one then the new distance is saved as variable (Dis) and new point coordinate saved as point $\mathrm{B}$. This process is continuing until the distance become larger than the saved distance. 


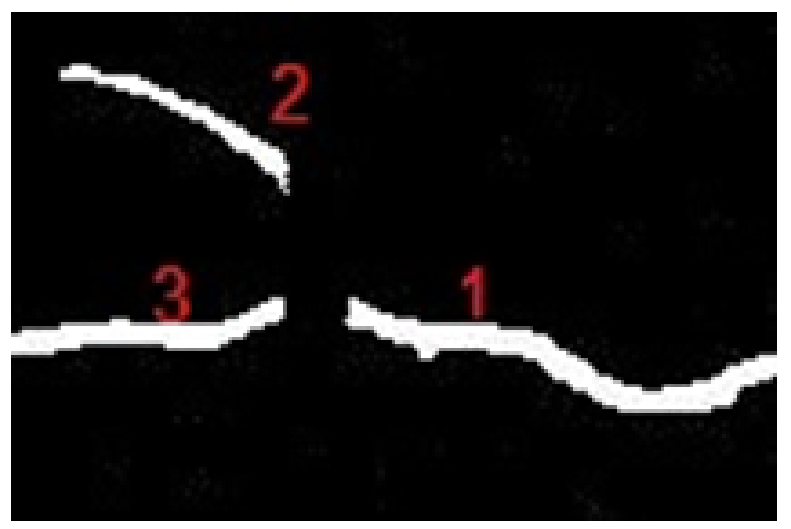

(A)

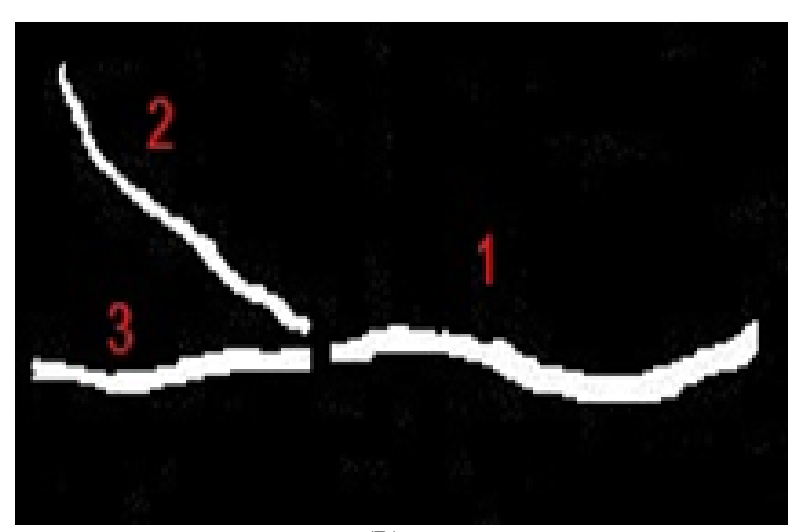

(B)

Fig. 6. (A) Blood vessels segments in healthy retinal (B) blood vessels segments in infected retinal

Table 1. Type of blood vessels according to its diameter

\begin{tabular}{ll}
\hline Diameter & Type of blood vessel \\
\hline Dis $\geq 11$ & Main vessel \\
$4 \leq$ Dis $\leq 7$ & Secondary \\
$2 \leq$ Dis $<3$ & Vascular \\
\hline
\end{tabular}

Otherwise if the distance at the first movement to the right was larger than the stored distance, then the direction of mask movement is changed to the left and the same processes are repeated in the left direction (Fig. 2) until we reach to the value of distance more than the saved value of distance.

The computed distance is representing the width (diameter) of the blood vessel segment. According to the value of blood vessel width we decide the type of blood vessel as in Table 1.

The suggested algorithm was tested, by applying it's on the suggested blood vessels with predefined its diameters as shown in the Fig. 5, the result was accurate and the three diameters determined accurately.

Also the algorithm tested on real images of retinal blood vessels network (healthy and infected retinal) as shown in Fig. 6. We tested the results according to Murray's law "The vessel network arrangement is based on Murray's law which states that the cube of the radius of a parent vessel equals the sum of the cube of the radii of the daughters" (Stefan, 2013).

In this test the accuracy of determining the blood vessels width were $98 \%$.

\section{CONCLUSION}

In this study we presented new way to determine the blood vessel diameter. The study suggested to creat new mask to help tracing the blood vessel, determine the edge (blood vessel wall) and ultimately measuring the blood vessel diameter. The algorithm tested with many suggested blood vessels (manually drawing) and the results were very accurate. Also the algorithm tested with real images for both healthy and infected retinal and with aided of Murray's law we found the accuracy about $98 \%$ which is better than many other algorithms. The suggested algorithm simple, fast and easy to applied.

\section{REFERENCES}

El Abbadi, N.K. and E.H. Al Saadi, 2013. Blood vessels extraction using mathematical morphology. J. Comput. Sci., 9: 1389-1395. DOI: 10.3844/jcssp.2013.1389.1395

Joshi, V.S., 2012. Analysis of retinal vessel networks using quantitative descriptors of vascular morphology. Ph.D. Thesis, University of Iowa, USA.

Klein, R., C.E. Myers, K.E. Lee, R. Gangnon and B.E. Klein, 2012. Changes in retinal vessel diameter and incidence and progression of diabetic retinopathy. Arch Ophthalmol., 130: 749-755. PMID: 22332203

Kumar, D.K., B. Aliahmad and H. Hao, 2012. Retinal vessel diameter measurement using unsupervised linear discriminant analysis. ISRN Ophthalmol.

Pakter, H.M., S.C. Fuchs, M.K. Maestri, L.B. Moreira and L.M. Dei Ricardi et al., 2011. Computerassisted methods to evaluate retinal vascular caliber: What are they measuring? invest. Ophthalmol. Visual Sci., 52: 810-815. PMID: 21051725 
Pemp, B., A.P. Cherecheanu G. Garhofer and L. Schmetterer, 2013. Calculation of central retinal artery diameters from non-invasive ocular haemodynamic measurements in type 1 diabetes patients. Acta Ophthalmol., 91: e348-e352. PMID: 23387954

Stefan, T., 2013. Characterization of retinal vessel networks in human retinal imagery using quantitative descriptors. Int. J. Biofl. Soc., 5: 52-57.
Xu, X., M. Niemeijer, Q. Song, M. Sonka and M.K. Garvin et al., 2011. Vessel boundary delineation on fundus images using graph-based approach. IEEE Trans. Med. Imag., 30: 1184-1191. PMID: 21216707 